\title{
Predictors of sinus rhythm after electrical cardioversion of atrial fibrillation: results from a data mining project on the Flec-SL trial data set
}

\author{
Emre Oto ${ }^{1}$, Sercan Okutucu ${ }^{2 *}$, Deniz Katircioglu-Öztürk ${ }^{1}$, Halil Altay Güvenir ${ }^{3}$, \\ Ergun Karaagaoglu ${ }^{4}$, Martin Borggrefe ${ }^{5}$, Günter Breithardt ${ }^{6,7}$, Andreas Goette ${ }^{6,8}$, \\ Ursula Ravens 9 , Gerhard Steinbeck ${ }^{\mathbf{1 0}}$, Karl Wegscheider ${ }^{11}$, Ali Oto ${ }^{2}$, and \\ Paulus Kirchhof $6,12,13$
}

\begin{abstract}
${ }^{1}$ Medical Information Technology Solutions (MITS), Bilkent University Cyberpark, Ankara, Turkey; ${ }^{2}$ Department of Cardiology, Memorial Ankara Hospital, Memorial Healthcare Group, Ankara, Turkey; ${ }^{3}$ Department of Computer Engineering, Faculty of Engineering, Bilkent University, Ankara, Turkey; ${ }^{4}$ Department of Biostatistics, Faculty of Medicine, Hacettepe University, Ankara, Turkey; ${ }^{5}$ Department of Cardiology, University of Mannheim, Mannheim, Germany; ${ }^{6}$ Atrial Fibrillation Network Association, Münster, Germany; ${ }^{7}$ Department of Cardiovascular Medicine, Division of Rhythmology, University Hospital Münster, Münster, Germany; ${ }^{8}$ Department of Cardiology, Vincenz-Krankenhaus, Paderborn, Germany; ${ }^{9}$ Department of Pharmacology, Technical University, Dresden, Germany; ${ }^{10}$ Department of Cardiology, Ludwig-Maximilians-University of Munich, Germany; ${ }^{11}$ Department of Medical Biometry and Epidemiology, University Medical Center Hamburg-Eppendorf, Hamburg, Germany; ${ }^{12}$ Institute of Cardiovascular Sciences, University of Birmingham and SWBH and UHB NHS Trusts, Birmingham, UK; and ${ }^{13}$ Department of Cardiovascular Medicine, University Hospital Münster, Münster, Germany
\end{abstract}

Received 4 February 2016; accepted after revision 27 April 2016; online publish-ahead-of-print 2 july 2016

Aims

Methods

and results

Conclusions

Keywords
Data mining is the computational process to obtain information from a data set and transform it for further use. Herein, through data mining with supportive statistical analyses, we identified and consolidated variables of the Flecainide ShortLong (Flec-SL-AFNET 3) trial dataset that are associated with the primary outcome of the trial, recurrence of persistent atrial fibrillation (AF) or death.

The 'Ranking Instances by Maximizing the Area under the ROC Curve' (RIMARC) algorithm was applied to build a classifier that can predict the primary outcome by using variables in the Flec-SL dataset. The primary outcome was time to persistent AF or death. The RIMARC algorithm calculated the predictive weights of each variable in the Flec-SL dataset for the primary outcome. Among the initial 21 parameters, 6 variables were identified by the RIMARC algorithm. In univariate Cox regression analysis of these variables, increased heart rate during AF and successful pharmacological conversion (PC) to sinus rhythm (SR) were found to be significant predictors. Multivariate Cox regression analysis revealed successful PC as the single relevant predictor of SR maintenance. The primary outcome risk was 3.14 times $(95 \%$ Cl:1.7-5.81) lower in those who had successful PC to SR than those who needed electrical cardioversion.

\section{Introduction}

Cardioversion of persistent and long-standing persistent atrial fibrillation (AF) is a common intervention in patients who are in need for rhythm control therapy. While this procedure restores sinus rhythm (SR) very effectively in the acute setting, ${ }^{1}$ long-term recurrences of AF are common after cardioversion. ${ }^{2,3}$ Identifying patients who are more likely to maintain SR for a longer time would help develop a personalized planning of rhythm control therapy. Unfortunately, such parameters are not well described. ${ }^{4}$

Data mining is the computational process of discovering patterns in large datasets involving methods at the intersection of artificial

* Corresponding author. Tel: +90 312 2536666; fax: +90 312 2536623. E-mail address: sercanokutucu@yahoo.com

Published on behalf of the European Society of Cardiology. All rights reserved. C The Author 2016. For permissions please email: journals.permissions@oup.com. 


\section{What's new?}

- Data mining is a novel computational process to obtain information from a data set and transform it for further use.

- In current study, through data mining with supportive statistical analyses, we identified and consolidated variables of the Flecainide Short-Long trial dataset that are associated with the primary outcome of the trial, recurrence of persistent atrial fibrillation or death.

- Pharmacological conversion of persistent AF with flecainide without the need for electrical cardioversion is a powerful and independent predictor of maintenance of sinus rhythm.

- A strategy of flecainide pretreatment for $48 \mathrm{~h}$ prior to planned electrical cardioversion may be a useful planning of a strategy of long-term rhythm control.

intelligence, machine learning, statistics, and database systems. The overall goal of the data mining process is to extract information from a data set and transform it into an understandable structure for further use. ${ }^{5-7}$ Aside from the raw analysis step, it involves database and data management aspects, data pre-processing, model and inference considerations, interestingness metrics, complexity considerations, post-processing of discovered structures, visualization, and online updating. ${ }^{5-7}$

The Flecainide Short-Long (Flec-SL-AFNET 3) trial $^{2,8}$ is a prospective, randomised, open-label, blinded endpoint assessment trial that evaluated whether short-term antiarrhythmic drug treatment was non-inferior to standard long-term treatment to prevent recurrent $A F$ in patients undergoing elective cardioversion. This trial revealed that short-term antiarrhythmic drug treatment after cardioversion was non-inferior to long-term treatment in a cohort of patients in whom electrical remodelling was thought to play a large part in recurrent $A F^{2}$ Additionally, the study confirmed that flecainide was effective in the prevention of recurrent $A F$ after cardioversion.

Here, we applied machine learning to determine predictors of long-term maintenance of SR in the Flec-SL-AFNET-3 data set. We used the RIMARC (Ranking Instances by Maximizing the Area under the ROC Curve) classification algorithm. ${ }^{5,6}$ This algorithm operates by ranking instances based on how likely they are to have a positive label indicating the presence of a condition. ${ }^{7}$ By means of this type of data mining analysis, we tried to extract clinically relevant statistical information from the Flec-SL-AFNET 3 database and seek for factors that might affect recurrence of persistent AF after cardioversion, the primary outcome of the trial. We then applied validation by biostatistical analysis.

\section{Methods}

\section{Study design and participants}

Between May 2007 and March 2010, 635 patients scheduled for planned electrical cardioversion were enrolled into a prospective, randomized, open-label, blinded endpoint assessment trial. ${ }^{8}$ Details of the trial design and the primary outcome have been reported previously. ${ }^{2}$ Eligible patients were adults with persistent $A F$ undergoing planned cardioversion. All patients received $48 \mathrm{~h}$ or more of treatment with the study drug flecainide before planned electrical cardioversion. This was a mandatory part of the protocol meant to ensure effective flecainide plasma levels at the time of cardioversion. After successful cardioversion, patients were randomly assigned in permuted blocks of six per center to: no antiarrhythmic drug treatment (control), treatment with flecainide for 4 weeks (short-term treatment) or flecainide for 6 months (long-term treatment). The primary outcome was time to persistent AF or death. ${ }^{2}$

\section{Data mining with RIMARC algorithm}

The major aim of pursuing a data mining experiment on the Flec-SL dataset was to extract clinically relevant information. As in almost all widely used data mining techniques, the main objective was to reveal underlying patterns in a data set and generate hypotheses over them, rather than describing the nature of the data and demonstrate the significance of a model that relates the data instances to the underlying population as conventional statistical methods mostly do. Data Mining employs such techniques as linear and non-linear classification, clustering and association algorithms to identify trends to be fed into further analyses. ${ }^{5-7}$ With this goal of using data mining for hypothesis and model generation, a supervised model based on the RIMARC (Ranking Instances by Maximizing the Area Under a (ROC) Curve) classification algorithm ${ }^{5}$ was constructed. In this scope, RIMARC algorithm was used to assign 'predictive weights', $\boldsymbol{w} \epsilon[0,1]$, to each of the baseline clinical parameter in the Flec-SL data set for addressing the 'class label' denoting whether a patient reaches the primary outcome or not.

Briefly, the RIMARC algorithm has been proven ${ }^{5}$ to constitute a ranking function that attains the maximal area under the ROC curve (AUC) for a given set of input parameters. The classification model learned by the RIMARC algorithm can be used to estimate the likelihood, score(q), of reaching the primary endpoint for any new patient $q$ as follows:

$$
\begin{gathered}
\text { score }(q)=\frac{\sum_{f} w_{f}^{q} \cdot s_{f}(q)}{\sum_{f} w_{f}^{q}}, \\
w_{f}^{q}=\left\{\begin{array}{lll}
w_{f} & q_{f} \text { is known } \\
0 & q_{f} \text { is missing }
\end{array}\right.
\end{gathered}
$$

where $w_{f}$ represents the weight of the clinical parameter (feature) $f, q_{f}$ is the value of the parameter (feature) $f$ for patient $q$, and $s_{f}(q)$ is the score associated with the value of the feature ffor patient $q .{ }^{5,6}$ RIMARC algorithm had been implemented as a proprietary suite in Java programming language and the source codes with executables are available upon request.

\section{Statistical analysis}

We used the RIMARC algorithm to individually identify the predictive power of parameters in predicting the primary outcome of the trial. For this analysis, we have only included the parameters that were collected at baseline and are biologically homogeneous. Full list can be seen in Table 1. As the univariate classifier for each parameter is built by RIMARC, the prediction accuracy of the calculated predictive weights (w) are assessed in terms of AUC and their significances are validated by the widely used 10-fold cross-validation technique. In 10-fold crossvalidation, the original sample is randomly partitioned into 10 equal size subsamples. Of the 10 subsamples, a single subsample is retained as the validation data for testing the model, and the remaining 9 subsamples are used as training data. The cross-validation process is then repeated 10 times (number of folds), with each of the 10 subsamples used exactly once as the validation data. The resulting risk classification was later used to calculate the AUC and the $95 \% \mathrm{Cl}$ corresponding to this AUC value. 


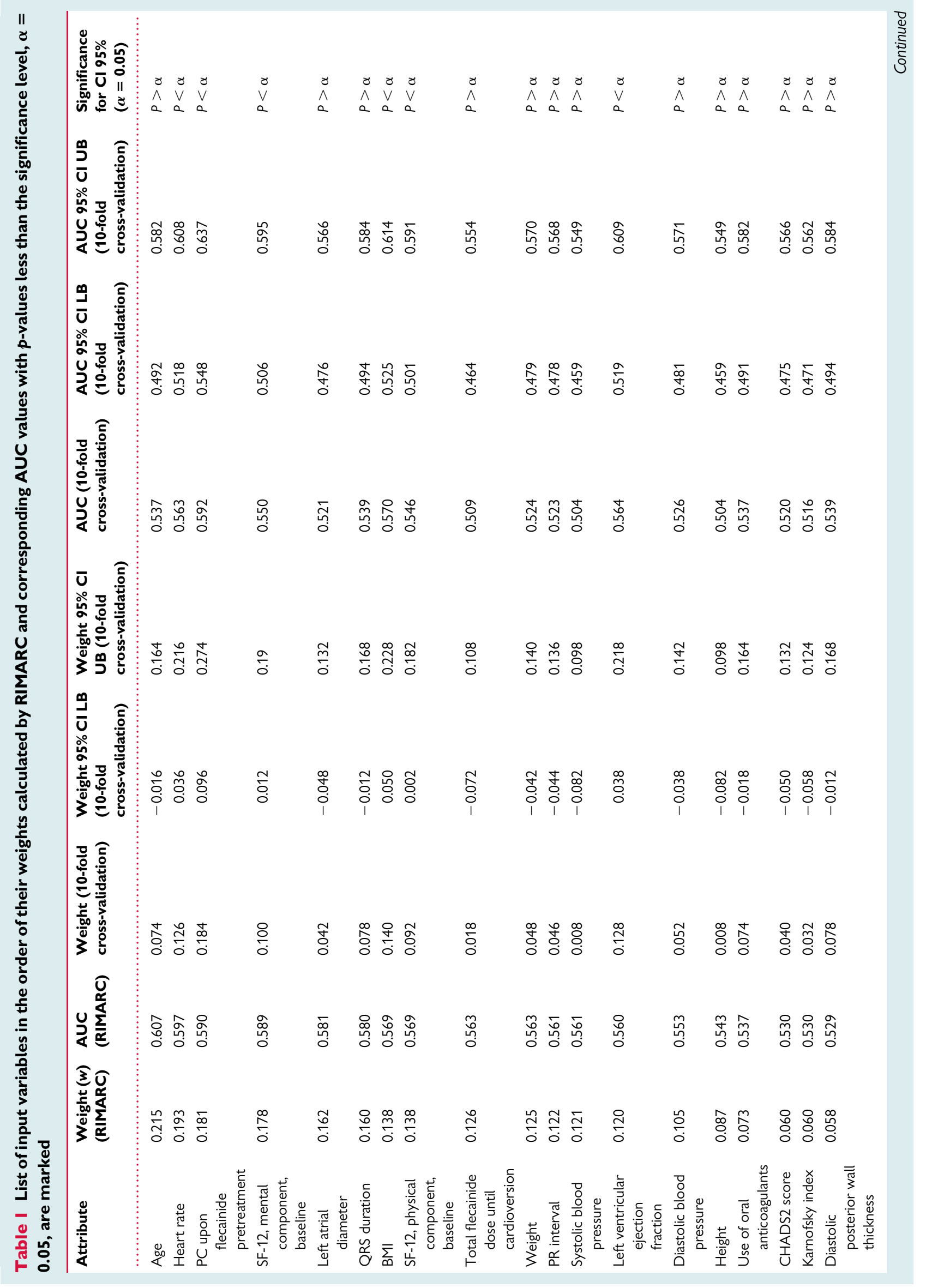




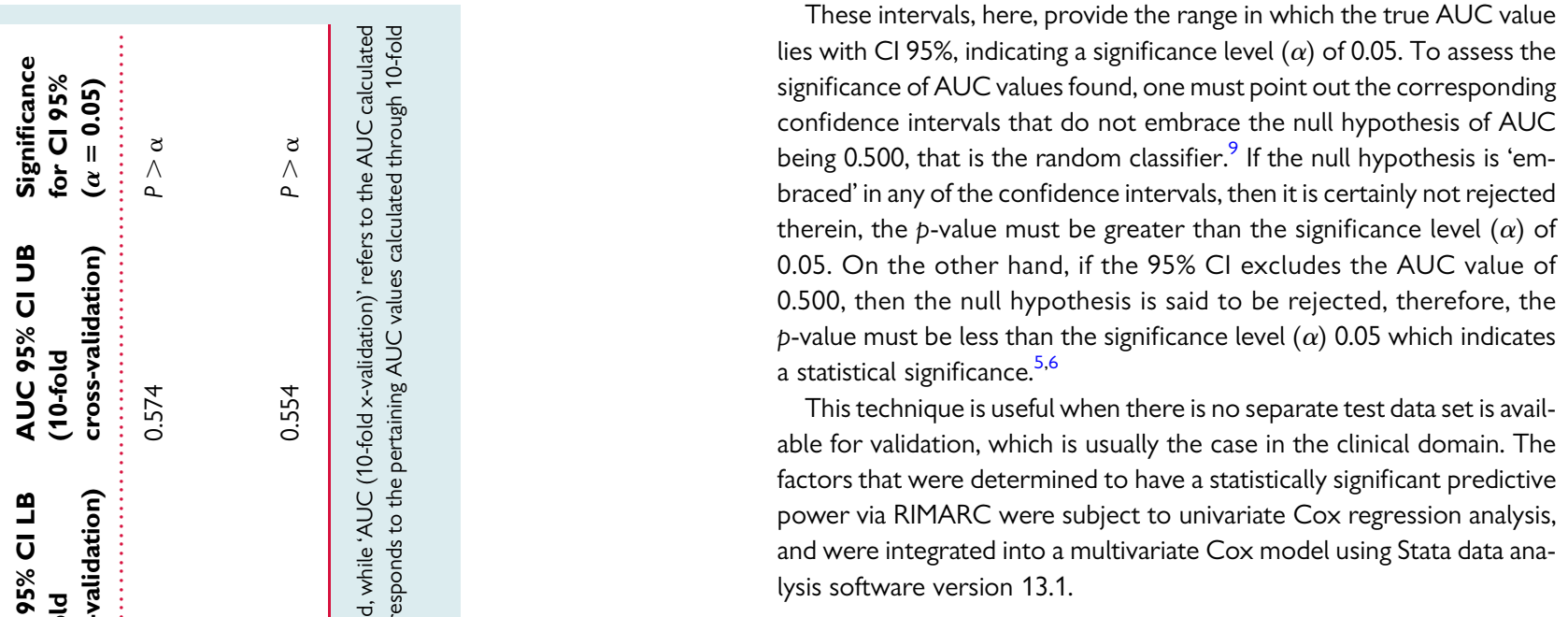

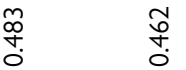

\section{Results}

\section{Variable selection and RIMARC-based classifiers}

By means of RIMARC algorithm, we obtained the predictive weights $(\mathbf{w})$ together with the AUC values of each baseline parameter associated with recurrence of $A F$ in other publications. ${ }^{4}$ After applying 10-fold cross-validation on the AUC values produced for each parameter, mean AUC's with $95 \%$ confidence intervals are calculated. Among the initial 21 parameters, 6 variables were identified by the RIMARC algorithm. These variables were heart rate, PC upon flecainide pretreatment, body mass index, left ventricular ejection fraction, baseline SF-12 physical, and mental component scores which indicated as having significant AUC values in determining the primary outcome (Table 1).

\section{Univariate and multivariate analysis}

The 6 parameters at baseline determined also to have a statistically significant discrimination power via RIMARC were subject to univariate Cox regression analysis and were then used to build a multivariate Cox model. Increased heart rate during AF and PC upon flecainide pretreatment were found to be the significant predictors (significance level, $\alpha=0.05$ ) in univariate Cox regression analysis. The results of these analyses are presented in Table 2.

It can be seen that the parameter of pharmacological conversion (PC) upon flecainide pretreatment is the single most relevant predictor of SR maintenance in multivariate Cox regression analysis. The results of these analyses are presented in Table 3. Multivariate Cox regression indicates that successful PC reduced the primary outcome risk by 3.14 times [ $95 \% \mathrm{Cl}: 1.7-5.81$ ] than those with need for electrical cardioversion (hazard ratio: $0.318[95 \% \mathrm{Cl}$ : $0.172-0.588] ; P<\alpha=0.05$ ).

As illustrated in Figure 1, the Flec-SL dataset also shows that while $51 \%$ of patients who need electrical cardioversion reach the primary endpoint, this proportion is $23 \%$ in those patients who undergo successful PC. Therefore, the dataset also indicates that patients who undergo successful PC are $2.22(P<\alpha=0.05)$ times less likely to reach the primary endpoint than those patients who need electrical cardioversion. 
Table 2 Univariate cox regression analysis

\begin{tabular}{|c|c|c|c|c|c|c|c|c|c|}
\hline & \multirow[t]{2}{*}{ B } & \multirow[t]{2}{*}{ SE } & \multirow[t]{2}{*}{$\begin{array}{l}\text { Sig. } \\
(\alpha=0.05)\end{array}$} & \multirow[t]{2}{*}{ HR } & \multicolumn{2}{|c|}{$\begin{array}{l}95.0 \% \mathrm{Cl} \text { for } \\
\mathrm{HR}\end{array}$} & \multicolumn{3}{|c|}{ Dependent variable: time to event (days) } \\
\hline & & & & & Lower & Upper & $\begin{array}{l}\text { Cases } \\
\text { available }\end{array}$ & $\begin{array}{l}\text { Cases } \\
\text { censored }\end{array}$ & $\begin{array}{l}\text { Missing } \\
\text { values }\end{array}$ \\
\hline Heart rate & -0.010 & 0.003 & 0.001 & 0.990 & 0.984 & 0.996 & 287 & 347 & 1 \\
\hline PC upon flecainide pretreatment & -1.033 & 0.196 & 0.000 & 0.356 & 0.242 & 0.523 & 287 & 348 & 0 \\
\hline BMI & 0.000 & 0.012 & 0.993 & 1.000 & 0.976 & 1.025 & 284 & 343 & 0 \\
\hline Left ventricular ejection fraction & 0.009 & 0.008 & 0.248 & 1.009 & 0.994 & 1.024 & 233 & 301 & 101 \\
\hline $\begin{array}{l}\text { SF-12, physical component, } \\
\text { baseline }\end{array}$ & -0.011 & 0.007 & 0.095 & 0.989 & 0.976 & 1.002 & 206 & 216 & 213 \\
\hline $\begin{array}{l}\text { SF-12, mental component, } \\
\text { baseline }\end{array}$ & 0.005 & 0.007 & 0.499 & 1.005 & 0.991 & 1.018 & 205 & 220 & 210 \\
\hline
\end{tabular}

BMI, body mass index; PC, pharmacological cardioversion.

Table 3 Multivariate cox regression analysis (full model)

\begin{tabular}{|c|c|c|c|c|c|c|}
\hline & \multirow[t]{2}{*}{ B } & \multirow[t]{2}{*}{ SE } & \multirow{2}{*}{$\begin{array}{l}\text { Sig. } \\
(\alpha=0.05)\end{array}$} & \multirow[t]{2}{*}{ HR } & \multicolumn{2}{|c|}{$95.0 \% \mathrm{Cl}$ for $\mathrm{HR}$} \\
\hline & & & & & Lower & Upper \\
\hline Heart rate & -0.005 & 0.004 & 0.269 & 0.995 & 0.987 & 1.004 \\
\hline PC upon flecainide pretreatment & -1.080 & 0.278 & 0.000 & 0.340 & 0.197 & 0.586 \\
\hline BMI & -0.003 & 0.016 & 0.864 & 0.997 & 0.966 & 1.029 \\
\hline Left ventricular ejection fraction & 0.013 & 0.009 & 0.171 & 1.013 & 0.995 & 1.031 \\
\hline SF-12, physical component, baseline & -0.010 & 0.008 & 0.178 & 0.990 & 0.975 & 1.005 \\
\hline \multirow[t]{2}{*}{ SF-12, mental component, baseline } & 0.001 & 0.008 & 0.925 & 1.001 & 0.986 & 1.016 \\
\hline & $N$ & $\%$ & & & & \\
\hline \multicolumn{7}{|l|}{ Cases available in analysis } \\
\hline Event $^{\mathrm{a}}$ & 172 & 27.1 & & & & \\
\hline Censored & 198 & 31.2 & & & & \\
\hline Total & 370 & 58.3 & & & & \\
\hline \multicolumn{7}{|l|}{ Cases dropped } \\
\hline Cases with missing values & 265 & 41.7 & & & & \\
\hline Cases with negative time & 0 & 0.0 & & & & \\
\hline Censored cases before the earliest event in a stratum & 0 & 0.0 & & & & \\
\hline Total & 265 & 41.7 & & & & \\
\hline Total & 635 & 100.0 & & & & \\
\hline
\end{tabular}

BMI, body mass index; PC, pharmacological cardioversion.

${ }^{a}$ Dependent variable: time to event (days).

The Kaplan-Meier survival analysis of the time-to-primary endpoint also indicates that the mean time-to-primary endpoint of patients who had successful PC is significantly higher than those patients who needed electrical cardioversion. Figure 2 illustrates the survival curves of patients who had successful PC and those who needed electrical cardioversion.

\section{Discussion}

Using a combination of explorative data mining analyses and confirmation by 'conventional' univariate and multivariate Cox hazard proportional models, we identified PC of persistent AF during inhospital flecainide initiation as an important predictor of longer term maintenance of SR after cardioversion of persistent AF. Patients who were cardioverted back to SR during oral pretreatment with flecainide for $48 \mathrm{~h}$ prior to scheduled electrical cardioversion were more than three times as likely to maintain SR for 6 months after cardioversion. Other clinical variables, including duration of $\mathrm{AF}$ and atrial size, were not predictive of recurrent $A F$ in this large data set of patients with persistent and long-standing persistent AF.

Several mechanisms cause recurrent AF after cardioversion. In addition to electrical remodelling, structural changes in the atria, 
focal firing from the pulmonary veins, and abnormal myocardial calcium handling can cause recurrences. ${ }^{10,11}$ Antiarrhythmic drugs such as flecainide prolong the action potential, increase postrepolarization refractoriness, and increase the curvature of electrical wave fronts in the left atrium, thereby contributing to termination of fibrillatory activity. In addition, flecainide can also prevent focal firing and abnormal intracellular calcium handling., 8,12

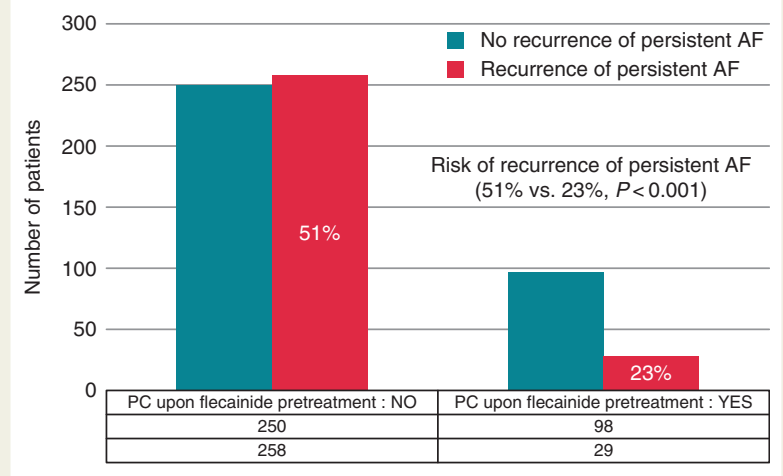

Figure I Distribution of patients who had recurrence of persistent $A F$ and who were in $S R$ (i.e. do not reach the primary endpoint) at the end of the 6-month follow-up period.
Pretreatment with flecainide prior to planned electrical cardioversion of AF has not been systematically evaluated before. Although therapeutic antiarrhythmic drug levels immediately after cardioversion can prevent immediate recurrences of AF, pretreatment does not routinely used in common clinical practice. Nonetheless, some observational data sets are available for patients pretreated with amiodarone ${ }^{13}$ and dofetilide ${ }^{14}$ : Galperín et al. ${ }^{13}$ analysed the role of different parameters that determine long-term SR maintenance in patients with persistent AF who are treated with amiodarone. They enrolled 141 anticoagulated patients with persistent AF who were pretreated for 4 weeks with oral amiodarone. Those in whom the arrhythmia persisted underwent electric cardioversion. After restoration of normal SR (either pharmacologic or electric), all patients received a daily dose of amiodarone and were followed $\sim 2$ years. Concordant with our findings, PC during pretreatment was one of the major determinants for long-term SR maintenance. $^{13}$

Malhotra et al. ${ }^{14}$ retrospectively reviewed elective inpatient admissions for dofetilide loading. They used a multivariate Cox proportional hazards model to assess predictors of maintenance of SR after in-hospital dofetilide loading. They found that patients who converted pharmacologically upon dofetilide pretreatment remained longer in SR compared with the patients who required electrical cardioversion. ${ }^{14}$ Thus, our main finding is in line with other reports on other antiarrhythmic drugs.

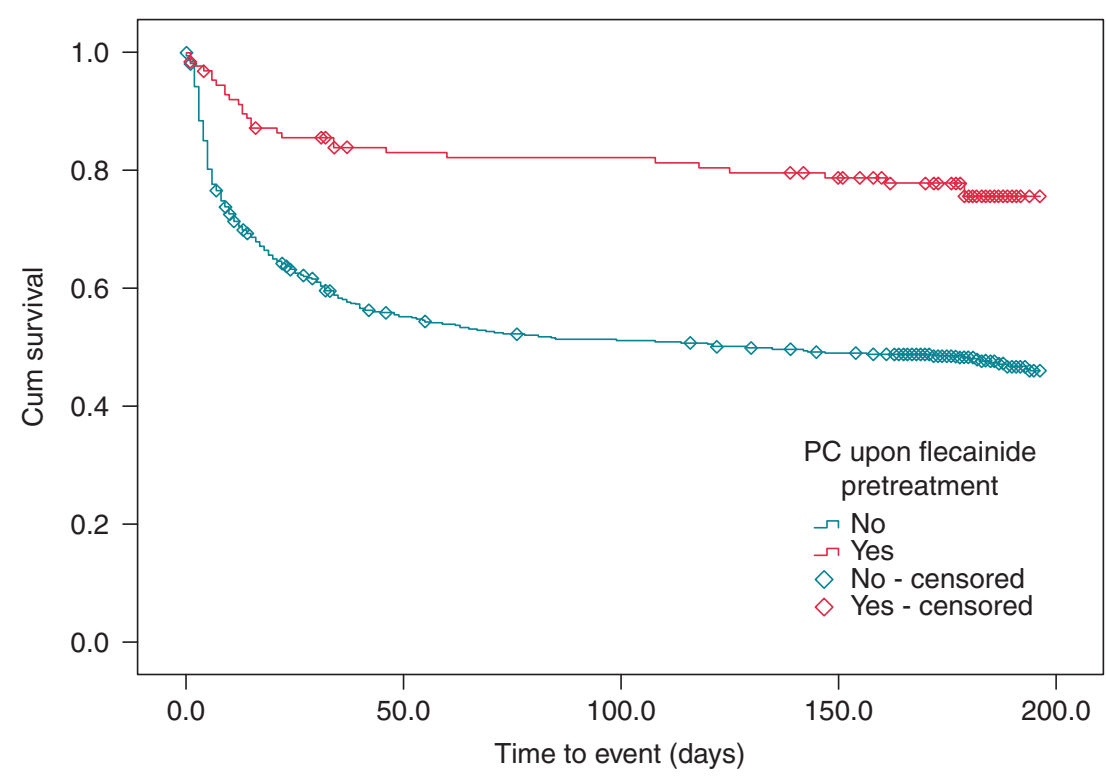

\begin{tabular}{|c|c|c|c|c|}
\hline \multirow{2}{*}{$\begin{array}{c}\text { PC upon } \\
\text { flecainide } \\
\text { pretreatment }\end{array}$} & \multicolumn{4}{|c|}{ MEAN } \\
\cline { 3 - 5 } & Estimate & Std. error & 95\% confidence interval \\
\cline { 4 - 5 } & & Lower bound & Upper bound \\
\hline NO & 106.941 & 4.047 & 99.008 & 114.873 \\
\hline YES & 161.149 & 6.239 & 148.921 & 173.377 \\
\hline Overall & 117.787 & 3.578 & 110.774 & 124.799 \\
\hline
\end{tabular}

Figure 2 Survival analysis comparing the time-to-primary-endpoint of patients who undergo successful PC and those who need electrical cardioversion. 
Recent epicardial mapping data suggest that the electrical complexity of AF differs markedly between patients with persistent $A F .{ }^{15,16}$ Although we cannot provide definite proof for this hypothesis, it is possible that $\mathrm{PC}$ of persistent AF during flecainide pretreatment could be a marker for low complexity of $A F .^{17,18}$ Furthermore, it is conceivable that PC of AF identifies a group of patients who are responsive to therapy with flecainide which was given to the majority of patients during follow-up. ${ }^{2}$

The main reason why RIMARC was selected as the data mining algorithm of choice is the proven accuracy and performance of RIMARC algorithm, and that it yields easily interpretable and clinically relevant results. The predictive performance of RIMARC algorithm over a wide range of commonly used data mining algorithms with 10 benchmark data sets was shown in comparative experiments. ${ }^{5}$ The comparison is made on the AUC metric owing to the fact that it is a widely accepted in evaluating the accuracy of a classification performance. Valid arguments were theoretically and empirically supplied as to why AUC should be preferred over accuracy, which is merely a ratio of correctly predicted results to the total number of instances examined. ${ }^{19,20} \mathrm{~A}$ higher value of AUC for a parameter is an indication of its higher relevance in determining the class label. Since RIMARC algorithm aims to maximize the AUC value directly, it outperforms the other data mining algorithms on average, with a statistically significant difference. ${ }^{5-7}$

Furthermore, RIMARC has several advantages compared with other algorithms of medical data mining and decision support, which can be summarized as follows:

(i) RIMARC is a non-parametric machine learning method, thus no parameter tuning is needed to attain an optimal classifier.

(ii) RIMARC is highly robust to the frequently encountered 'missing value' problem in clinical datasets.

(iii) RIMARC's main output is a human-readable, intuitive 'ranking function' that lists the order of influence of input variables and their impacts (i.e.'weights') on the ranking function.

(iv) The impacts of individual input variables on the ranking function are calculated independently. Therefore, any addition or removal of variables does not affect the individual weights of the input variables in the classifier. ${ }^{5,6}$

\section{Limitations}

The present analysis is a retrospective analysis of the Flec-SL-AFNET 3 data set. The data set provides information on a large contemporary cohort of patients undergoing cardioversion of AF. In addition, the data mining used, where the hypothesis is not prespecified, and the study is initially hypothesis free. As any retrospective analysis, it is hypothesis generating. This is a large cohort of patients, many of whom have long-standing AF (mean duration 28 months), therefore spontaneous conversions occurred in $\sim 20 \%$ of patients.

\section{Conclusion and clinical perspective}

This analysis of the Flec SL-AFNET 3 data set using both data mining and classical statistical methods suggests that PC during $48 \mathrm{~h}$ of oral therapy of persistent AF patients with flecainide is a powerful and independent predictor of maintenance of SR after cardioversion. Based on these findings, some additional insight into the durability of SR after cardioversion and AF treatment is obtained. Collection of further data is needed to establish and plan subsequent steps in short-term, longterm antiarrhythmic drug treatment and prevent recurrent AF after cardioversion. Acknowledging the hypothesis-generating nature of our analysis, oral pretreatment with antiarrhythmic drugs seems a reasonable and simple intervention prior to cardioversion that can help to identify patients at low risk of recurrent AF.

\section{Funding}

This work was supported by European Union through EUTRAF (FP7, to P.K., A.G., and A.O.) and under grant agreement No 633193 (CATCH ME, to P.K.), by British Heart Foundation (FS/13/32/30324, to P.K.), by the German Ministry of Education and Research through AFNET (01GI0204, to G.B., U.R., A.G., P.K.) and through funds from the German Centre for Cardiovascular Research (DZHK, to P.K. and G.B.). Additional funds for the conduct of the FLec SL-AFNET 3 trial were provided by MEDA Pharma.

Conflict of interest: none declared.

\section{References}

1. Kirchhof P, Mönnig G, Wasmer K, Heinecke A, Breithardt G, Eckardt L et al. A trial of self-adhesive patch electrodes and hand-held paddle electrodes for external cardioversion of atrial fibrillation (MOBIPAPA). Eur Heart J 2005;26: 1292-7.

2. Kirchhof P, Andresen D, Bosch R, Borggrefe M, Meinertz T, Parade $U$ et al. Shortterm versus long-term antiarrhythmic drug treatment after cardioversion of atrial fibrillation (Flec-SL): a prospective, randomised, open-label, blinded endpoint assessment trial. Lancet 2012;380:238-46.

3. Camm AJ, Lip GY, De Caterina R, Savelieva I, Atar D, Hohnloser SH et al. 2012 focused update of the ESC Guidelines for the management of atrial fibrillation: an update of the 2010 ESC Guidelines for the management of atrial fibrillation developed with the special contribution of the European Heart Rhythm Association. Europace 2012;14:1385-413.

4. Kirchhof P, Lip GY, Van Gelder IC, Bax J, Hylek E, Kaab S et al. Comprehensive risk reduction in patients with atrial fibrillation: emerging diagnostic and therapeutic options - a report from the 3rd Atrial Fibrillation Competence NETwork/ European Heart Rhythm Association consensus conference. Europace 2012;14: $8-27$.

5. Guvenir HA, Kurtcephe M. Ranking instances by maximizing the area under ROC curve. IEEE Trans Knowledge Data Eng 2013;25:2356-66.

6. Guvenir HA, Kurtcephe M. A discretization method based on maximizing the area under receiver operating characteristic curve. Int J Pattern Recogn Artif Intell 2013;27: 1350002.

7. Ravens U, Katircioglu-Ozturk D, Wettwer E, Christ T, Dobrev D, Voigt N et al. Application of the RIMARC algorithm to a large data set of action potentials and clinical parameters for risk prediction of atrial fibrillation. Med Biol Eng Comput 2015; 53:263-73.

8. Kirchhof P, Fetsch T, Hanrath P, Meinertz T, Steinbeck G, Lehmacher W et al Targeted pharmacological reversal of electrical remodeling after cardioversionrationale and design of the Flecainide Short-Long (Flec-SL) trial. Am Heart J 2005; 150:899, e1-e6.

9. du Prel JB, Hommel G, Rohrig B, Blettner M. Confidence interval or p-value?: part 4 of a series on evaluation of scientific publications. Dtsch Arztebl Int 2009; 106:335-9.

10. Andrade JG, Nattel S, Macle L. Late pulmonary vein reconnection: insights into mechanisms of atrial fibrillation recurrence after pulmonary vein isolation. Europace 2013;15:1143.

11. Manios EG, Kanoupakis EM, Chlouverakis GI, Kaleboubas MD, Mavrakis HE, Vardas PE. Changes in atrial electrical properties following cardioversion of chronic atrial fibrillation: relation with recurrence. Cardiovasc Res 2000;47: 244-53.

12. Kirchhof P, Engelen M, Franz MR, Ribbing M, Wasmer K, Breithardt G et al. Electrophysiological effects of flecainide and sotalol in the human atrium during persistent atrial fibrillation. Basic Res Cardiol 2005;100:112-21.

13. Galperin J, Elizari MV, Chiale PA, Molina RT, Ledesma R, Scapin AO et al. Pharmacologic reversion of persistent atrial fibrillation with amiodarone predicts long-term sinus rhythm maintenance. J Cardiovasc Pharmacol Ther 2003;8:179-86. 
14. Malhotra R, Bilchick KC, DiMarco JP. Usefulness of pharmacologic conversion of atrial fibrillation during dofetilide loading without the need for electrical cardioversion to predict durable response to therapy. Am J Cardiol 2014;113:475-9.

15. de Groot NM, Houben RP, Smeets JL, Boersma E, Schotten U, Schalij MJ et al. Electropathological substrate of longstanding persistent atrial fibrillation in patients with structural heart disease: epicardial breakthrough. Circulation 2010;122: 1674-82.

16. Allessie MA, de Groot NM, Houben RP, Schotten U, Boersma E, Smeets JL et al. Electropathological substrate of long-standing persistent atrial fibrillation in patients with structural heart disease: longitudinal dissociation. Circ Arrhythmia Electrophysiol 2010;3:606-15.
17. Wijffels MC, Dorland R, Mast F, Allessie MA. Widening of the excitable gap during pharmacological cardioversion of atrial fibrillation in the goat: effects of cibenzoline, hydroquinidine, flecainide, and d-sotalol. Circulation 2000;102:260-7.

18. Danse PW, Garratt C], Allessie MA. Flecainide widens the excitable gap at pivot points of premature turning wavefronts in rabbit ventricular myocardium. J Cardiovasc Electrophysiol 2001;12:1010-7.

19. Cook NR. Use and misuse of the receiver operating characteristic curve in risk prediction. Circulation 2007;115:928-35.

20. Okutucu S, Katircioglu-Öztürk D, Oto E, Güvenir HA, Karaagaoglu E, Oto A et al. Data mining experiments on the Angiotensin II-Antagonist in Paroxysmal Atrial Fibrillation (ANTIPAF-AFNET 2) trial: 'exposing the invisible'. Europace 2017:19:741-6.

\section{EP CASE EXPRESS}

doi:10.1093/europace/euw183

\section{Sinkhole syncope}

\section{Sarah Gutman* and Stuart Moir}

Monash Cardiovascular Research Centre, Monash HEART, Monash Health and Department of Medicine (MMC), Monash University, Melbourne, Australia

* Corresponding author. E-mail address: sarahgutman@gmail.com

A 53-year-old woman presented in complete heart block after an episode of syncope. She was hanging out washing when a $5 \mathrm{~m}$ deep sinkhole opened up beneath her.

On arrival to our emergency department, the patient was lucid and asymptomatic. The clinical examination was unremarkable.

Twelve-lead electrocardiogram showed sinus rhythm with thirddegree atrioventricular (AV) block and a junctional escape of 50 bpm (Panel A). Initial investigations were unremarkable.

Transthoracic echocardiogram demonstrated severely reduced left ventricular (LV) ejection fraction with akinesis of all mid-myocardial segments extending into adjacent basal and apical segments but sparing true apex and the very base (Panel $B$ ).

Three hundred and twenty-slice computed tomography coronary angiogram demonstrated no coronary atherosclerosis (Panel C), confirming the clinical suspicion that the patient's abnormal LV contraction represented a stress cardiomyopathy.

The patient remained in complete heart block with a stable junctional escape rhythm (40-50 bpm). Seven days post-admission, a repeat transthoracic echocardiogram demonstrated normalization of LV contraction (Panel D), yet complete heart block persisted. A dualchamber permanent pacemaker was inserted.

There have been rare reports of high-degree AV block and takotsubo cardiomyopathy occurring together in medical literature. Occasionally, cardiomyopathy persists after improvement of left ventricular wall motion necessitating implantation of a permanent pacemaker.

The full-length version of this report can be viewed at: http://www. escardio.org/Guidelines-\&-Education/E-learning/Clinical-cases/Electrophysiology/EP-Case-Reports.
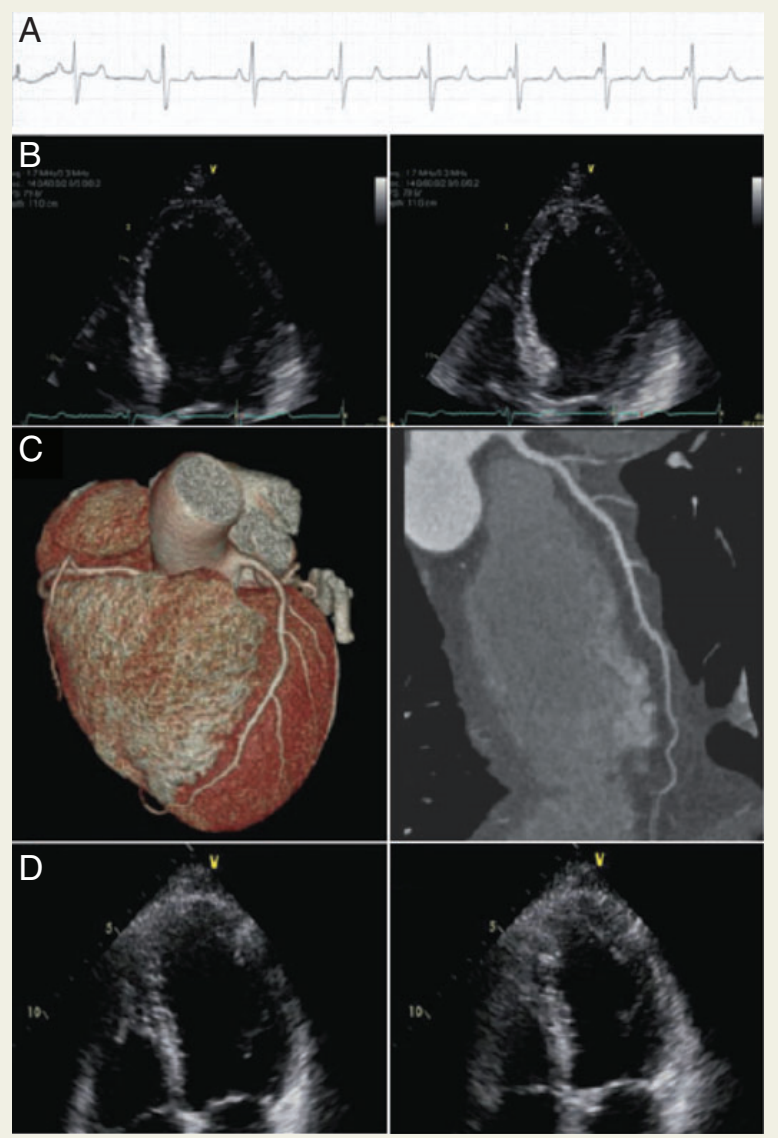